\title{
Null controllability of the heat equation using flatness
}

\author{
Philippe Martin $^{\mathrm{a}}$, Lionel Rosier ${ }^{\mathrm{b}}$, Pierre Rouchon ${ }^{\mathrm{a}}$ \\ ${ }^{a}$ Centre Automatique et Systèmes, MINES ParisTech, 60 boulevard Saint-Michel 75272 Paris Cedex 06, France \\ ${ }^{\mathrm{b}}$ Institut Élie Cartan de Lorraine, UMR 7502 UdL/CNRS/INRIA, BP 70239, 54506 Vandoeuvre-lès-Nancy, France
}

\begin{abstract}
We derive in a direct and rather straightforward way the null controllability of the $N$-dimensional heat equation in a bounded cylinder with boundary control at one end of the cylinder. We use the so-called flatness approach, which consists in parameterizing the solution and the control by the derivatives of a "flat output". This yields an explicit control law achieving the exact steering to zero. Replacing the involved series by partial sums we obtain a simple numerical scheme for which we give explicit error bounds. Numerical experiments demonstrate the relevance of the approach.
\end{abstract}

Key words: Partial differential equations; heat equation; boundary control; null controllability; motion planning; flatness.

\section{Introduction}

The controllability of the heat equation was first considered in the 1 -D case $[4,17,11,15]$ and very precise results were obtained by the classical moment approach. Next using Carleman estimates and duality arguments the null controllability was proved in $[7,13]$ for any bounded domain in $\mathbb{R}^{N}$, any control time $T$, and any control region. This Carleman approach proves very efficient also with semilinear parabolic equations [7]. By contrast the interest for the numerical investigation of the null controllability of the heat equation (or of parabolic equations) is fairly recent: apart from [3], the first significant contributions are $[32,31,24,2,23,1,5]$; see also [8] for an application to some inverse problems. All the above results rely on some observability inequalities for the adjoint system. A direct approach which does not involve the adjoint problem was proposed in $[11,15,14,16]$. In [11] a fundamental solution for the heat equation with compact support in time was introduced and used to prove null controllability. The results in $[11,27]$ can be used to derive control results on a bounded interval with two or one boundary control in some Gevrey class, or on a bounded domain of $\mathbb{R}^{N}$ with a control supported on the whole boundary (see also [16]). An extension of those results to the semilinear heat equation in 1-D was obtained in [14] in a more explicit way through the resolution of

Email addresses: philippe.martin@mines-paristech.fr (Philippe Martin), Lionel.Rosier@univ-lorraine.fr (Lionel Rosier), pierre.rouchon@mines-paristech.fr (Pierre Rouchon). an ill-posed problem with data of Gevrey order 2 in $t$.

In this paper, which builds on the preliminary versions $[19,20]$, we derive in a straightforward way the null controllability of the heat equation in a bounded cylinder $\Omega=\omega \times(0,1) \subset \mathbb{R}^{N}$ with Neumann boundary control on $\omega \times\{1\}$. More precisely given any final time $T>0$ and initial state $\theta_{0} \in L^{2}(\Omega)$ we provide an explicit and very regular control such that the state reached at time $T$ is exactly zero. We use the so-called flatness approach [6], which consists in parameterizing the solution $\theta$ and the control $u$ by the derivatives of a "flat output" $y$; this notion was initially introduced for finite-dimensional (nonlinear) systems, and later extended to (in particular) parabolic 1-dimensional PDEs $[12,18,22,21]$. Choosing a suitable trajectory for this flat output $y$ then yields an explicit series for a control achieving the exact steering to zero. Note this paper is probably the first example of using flatness for the motion planning of a "truly" $N$-dimensional PDE. Comparing our results to $[14,16]$ we note that: (i) our control is not supported on the whole boundary even in dimension $N>1$; (ii) the control and the solution are Gevrey of order $s \in(1,2)$ in time; (iii) the control and the solution are developed in series whose easily computed partial sums yield accurate numerical approximations of both the control and the solution.

The paper runs as follows. In section 3 we consider the control problem in dimension $N=1$. In Proposition 1 we investigate an ill-posed problem with Cauchy data in a Gevrey class and prove its (global) well-posedness. 
Theorem 3 then establishes the null controllability in small time for any initial data in $L^{2}$. In section 4 we extend these results to the cylinder $\Omega=\omega \times(0,1) \subset$ $\mathbb{R}^{N}$. Section 5 provides accurate error estimates when the various series involved are replaced by their partial sums. Finally in section 6 some numerical experiments demonstrate the interest of the approach.

\section{Preliminaries (Gevrey functions)}

In the sequel we consider series with infinitely many derivatives of functions. The notion of Gevrey order is a way of estimating the growth of these derivatives: we say that a function $y \in C^{\infty}([0, T])$ is Gevrey of order $s \geq 0$ on $[0, T]$ if there exist positive constants $M, R$ such that

$$
\left|y^{(p)}(t)\right| \leq M \frac{p !^{s}}{R^{p}} \quad \forall t \in[0, T], \forall p \geq 0
$$

More generally if $K \subset \mathbb{R}^{N}$ is a compact set and $y$ is a function of class $C^{\infty}$ on $K$ (i.e. $y$ is the restriction to $K$ of a function of class $C^{\infty}$ on some open neighbourhood $\Omega$ of $K)$, we say $y$ is Gevrey of order $s_{1}$ in $x_{1}, s_{2}$ in $x_{2}, \ldots, s_{N}$ in $x_{N}$ on $K$ if there exist positive constants $M, R_{1}, \ldots, R_{N}$ such that $\forall x \in K, \forall p \in \mathbb{N}^{N}$

$$
\left|\partial_{x_{1}}^{p_{1}} \partial_{x_{2}}^{p_{2}} \cdots \partial_{x_{N}}^{p_{N}} y(x)\right| \leq M \frac{\prod_{i=1}^{N}\left(p_{i} !\right)^{s_{i}}}{\prod_{i=1}^{N} R_{i}^{p_{i}}} .
$$

By definition, a Gevrey function of order $s$ is also of order $r$ for $r \geq s$. Gevrey functions of order 1 are analytic (entire if $s<1$ ). Gevrey functions of order $s>1$ have a divergent Taylor expansion; the larger $s$, the "more divergent" the Taylor expansion. Important properties of analytic functions generalize to Gevrey functions of order $s>1$ : the scaling, addition, multiplication and derivation of Gevrey functions of order $s>1$ is of order $s$, see $[25,28,30]$. But contrary to analytic functions, Gevrey functions of order $s>1$ may be constant on an open set without being constant everywhere. For example the "step function"

$$
\phi_{s}(t):= \begin{cases}1 & \text { if } t \leq 0 \\ 0 & \text { if } t \geq 1 \\ \frac{e^{-(1-t)^{-k}}}{e^{-(1-t)^{-k}}+e^{-t^{-k}}} & \text { if } t \in(0,1)\end{cases}
$$

where $k=(s-1)^{-1}$ is Gevrey of order $s$ on $[0,1]$ (and in fact on $\mathbb{R})$; notice $\phi_{s}(0)=1, \phi_{s}(1)=0$ and $\phi_{s}^{(i)}(0)=$ $\phi_{s}^{(i)}(1)=0$ for all $i \geq 1$.

In conjunction with growth estimates we will repeatedly use Stirling's formula $n ! \sim(n / e)^{n} \sqrt{2 \pi n}$.

\section{The one-dimensional heat equation}

For simplicity we first study the 1-D heat equation

$$
\begin{aligned}
\partial_{t} \theta(t, x)-\partial_{x}^{2} \theta(t, x) & =0, & & (t, x) \in(0, T) \times(0,1) \\
\partial_{x} \theta(t, 0) & =0, & & t \in(0, T) \\
\partial_{x} \theta(t, 1) & =u(t), & & t \in(0, T)
\end{aligned}
$$

with initial condition in $L^{2}(0,1)$

$$
\theta(0, x)=\theta_{0}(x), \quad x \in(0,1)
$$

We claim the system (1)-(3) is "flat" with $y(t):=\theta(t, 0)$ as a flat output, meaning there is (in appropriate spaces of smooth functions) a $1-1$ correspondence between arbitrary functions $t \mapsto y(t)$ and solutions of (1)-(3).

We first seek a formal solution in the form

$$
\theta(t, x):=\sum_{i \geq 0} \frac{x^{i}}{i !} a_{i}(t)
$$

where the $a_{i}$ 's are functions yet to define. Plugging this expression into (1) yields

$$
\sum_{i \geq 0} \frac{x^{i}}{i !}\left[a_{i+2}-a_{i}^{\prime}\right]=0
$$

hence $a_{i+2}=a_{i}^{\prime}$ for all $i \geq 0$. On the other hand $y(t)=$ $\theta(t, 0)=a_{0}(t)$, and $(2)$ implies $a_{1}(t)=0$. As a consequence $a_{2 i}=y^{(i)}$ and $a_{2 i+1}=0$ for all $i \geq 0$. The formal solution thus reads

$$
\theta(t, x)=\sum_{i \geq 0} \frac{x^{2 i}}{(2 i) !} y^{(i)}(t)
$$

while the formal control is given by

$$
u(t)=\theta_{x}(t, 1)=\sum_{i \geq 1} \frac{y^{(i)}(t)}{(2 i-1) !}
$$

We now give a meaning to this formal solution by restricting $t \mapsto y(t)$ to be Gevrey of order $s \in[0,2)$.

Proposition 1 Let $s \in[0,2),-\infty<t_{1}<t_{2}<\infty$, and $y \in C^{\infty}\left(\left[t_{1}, t_{2}\right]\right)$ satisfying for some constants $M, R>0$

$$
\left|y^{(i)}(t)\right| \leq M \frac{i !^{s}}{R^{i}}, \quad \forall i \geq 0, \forall t \in\left[t_{1}, t_{2}\right]
$$

Then the function $\theta$ defined by (4) is Gevrey of order $s$ in $t$ and $s / 2$ in $x$ on $\left[t_{1}, t_{2}\right] \times[0,1]$; hence the control $u$ defined by (5) is also Gevrey of order $s$ on $\left[t_{1}, t_{2}\right]$. 
PROOF. We must prove the formal series

$$
\partial_{t}^{m} \partial_{x}^{n} \theta(t, x)=\sum_{2 i \geq n} \frac{x^{2 i-n}}{(2 i-n) !} y^{(i+m)}(t)
$$

is uniformly convergent on $\left[t_{1}, t_{2}\right] \times[0,1]$ with growth estimates of the form

$$
\left|\partial_{t}^{m} \partial_{x}^{n} \theta(t, x)\right| \leq C \frac{m !^{s}}{R_{1}^{m}} \frac{n !^{\frac{s}{2}}}{R_{2}^{n}} .
$$

By (6), we have for all $(t, x) \in\left[t_{1}, t_{2}\right] \times[0,1]$

$$
\begin{aligned}
\left|\frac{x^{2 i-n}}{(2 i-n) !} y^{(i+m)}(t)\right| & \leq \frac{M}{R^{i+m}} \frac{(i+m) !^{s}}{(2 i-n) !} \\
& \leq \frac{M}{R^{i+m}} \frac{\left(2^{i+m} i ! m !\right)^{s}}{(2 i-n) !} \\
& \leq \frac{M^{\prime}}{R^{i+m}} \frac{2^{s i}\left(2^{-2 i} \sqrt{\pi i}(2 i) !\right)^{\frac{s}{2}}}{(2 i-n) !} \frac{m !^{s}}{2^{-s m}} \\
& \leq M^{\prime} \frac{(\pi i)^{\frac{s}{4}}}{R_{1}^{i}(2 i-n) !^{1-\frac{s}{2}}} n !^{\frac{s}{2}} \frac{m !^{s}}{R_{1}^{m}}
\end{aligned}
$$

where we have set $R_{1}=2^{-s} R$ and $M^{\prime} \geq M$ is a suitable constant; we have used Stirling's formula for $(2 i)$ ! and twice $(i+j) ! \leq 2^{i+j} i ! j !$. Since $\sum_{2 i \geq n} \frac{(\pi i)^{\frac{s}{4}}}{R_{1}^{i}(2 i-n) !^{1-\frac{s}{2}}}<\infty$ the series in (7) are uniformly convergent for all $m, n \geq 0$, hence $\theta \in C^{\infty}\left(\left[t_{1}, t_{2}\right] \times[0,1]\right)$. Finally, since

$$
\sum_{2 i \geq n} \frac{M^{\prime}(\pi i)^{\frac{s}{4}}}{R_{1}^{i}(2 i-n) !^{1-\frac{s}{2}}} \leq \frac{M^{\prime}}{R_{1}^{\frac{n}{2}}}\left(\frac{\pi}{2}\right)^{\frac{s}{4}} \sum_{j \geq 0} \frac{j^{\frac{s}{4}}+n^{\frac{s}{4}}}{R_{1}^{\frac{j}{2}} j !^{1-\frac{s}{2}}} \leq C R_{2}^{-n}
$$

where $R_{2} \in\left(0, \sqrt{R_{1}}\right)$ and $C>0$ is some constant independent of $n$, we have the desired estimates (8).

Remark 2 Note the Cauchy-Kovalevsky theorem, see e.g. [10], only ensures for $y \in C^{\omega}(0, T)$ the existence of solutions of the Cauchy problem composed of (1)-(2) and $\theta(t, 0)=y(t)$ on a small neighborhood of $\{x=0\}$; whereas we are interested in non-analytic solutions $\theta$ defined for all $(t, x) \in[0, T] \times[0,1]$.

We now derive an explicit control steering the system from any initial state $\theta_{0} \in L^{2}(0,1)$ at time 0 to the final state 0 at time $T>0$. Two ideas are involved: on the one hand thanks to the flatness property it is easy to find a control achieving the steering to zero starting from a certain set of initial conditions (step 2 in the proof of Theorem 3); on the other hand thanks to the regularizing property of the heat equation this set is reached from any $\theta_{0} \in L^{2}(0,1)$ when applying first a zero control for some time (step 1 in the proof of Theorem 3 ).

Theorem 3 Let $\theta_{0} \in L^{2}(0,1), T>0, \tau \in(0, T)$ and $s \in(1,2)$. Then there exists a function $y:[\tau, T] \rightarrow \mathbb{R}$
Gevrey of order s on $[\tau, T]$ such that the control

$$
u(t):= \begin{cases}0 & \text { if } 0 \leq t \leq \tau \\ \sum_{i \geq 1} \frac{y^{(i)}(t)}{(2 i-1) !} & \text { if } \tau<t \leq T\end{cases}
$$

steers the system (1)-(3) from the initial state $\theta(0,)=.\theta_{0}$ at time 0 to the final state $\theta(T,)=$.0 at time $T$.

Moreover $u$ is Gevrey of order s on $[0, T]$; the solution $\theta$ of (1)-(3) is Gevrey of order s int and s/2 in $x$ on $[\varepsilon, T] \times$ $[0,1]$ for all $\varepsilon \in(0, T)$.

PROOF. We first apply a null control on $[0, \tau]$ to reach a regular intermediate state (step 1), and then use the flatness property on $[\tau, T]$ to steer this intermediate state to 0 (step 2).

Step 1: Free evolution Let $u(t)=0$ for $t \in[0, \tau]$. Decompose $\theta_{0}$ as the Fourier series of cosines

$$
\theta_{0}(x)=\sum_{n \geq 0} c_{n} \sqrt{2} \cos (n \pi x)
$$

where the convergence holds in $L^{2}(0,1)$ and

$$
2\left|c_{0}\right|^{2}+\sum_{n \geq 1}\left|c_{n}\right|^{2}=\int_{0}^{1}\left|\theta_{0}(x)\right|^{2} d x<\infty .
$$

The solution starting from $\theta_{0}$ then reads

$$
\theta(t, x)=\sum_{n \geq 0} c_{n} e^{-n^{2} \pi^{2} t} \sqrt{2} \cos (n \pi x)
$$

and in particular

$$
\theta_{\tau}(x):=\theta(\tau, x)=\sum_{n \geq 0} c_{n} e^{-n^{2} \pi^{2} \tau} \sqrt{2} \cos (n \pi x) .
$$

Lemma $4 \theta_{\tau}$ is analytic in $\mathbb{C}$ and can be expanded as

$$
\theta_{\tau}(x)=\sum_{i \geq 0} y_{i} \frac{x^{2 i}}{(2 i) !}
$$

with

$$
\left|y_{i}\right| \leq C\left(1+\frac{1}{\sqrt{\tau}}\right) \frac{i !}{\tau^{i}}
$$

and $0<C \leq K \sup _{n \geq 0}\left|c_{n}\right|$, where $K$ is a universal positive constant.

Proof of Lemma 4: $\theta_{\tau}$ is analytic in $\mathbb{C}$ since for $|x| \leq r$

$$
\left|c_{n} e^{-n^{2} \pi^{2} \tau} \sqrt{2} \cos (n \pi x)\right| \leq C e^{-n^{2} \pi^{2} \tau+n \pi r},
$$


which ensures the uniform convergence of the series (10) in every open disk of radius $r>0$. On the other hand

$$
\begin{aligned}
\theta_{\tau}(x) & =\sqrt{2} \sum_{n \geq 0} c_{n} e^{-n^{2} \pi^{2} \tau} \sum_{i \geq 0}(-1)^{i} \frac{(n \pi x)^{2 i}}{(2 i) !} \\
& =\sum_{i \geq 0} \frac{x^{2 i}}{(2 i) !} \underbrace{\left(\sqrt{2} \sum_{n \geq 0} c_{n} e^{-n^{2} \pi^{2} \tau}\left(-n^{2} \pi^{2}\right)^{i}\right)}_{=: y_{i}} .
\end{aligned}
$$

The change in the order of summation will be justified once proved that

$$
\sum_{i, n \geq 0} \frac{(n \pi x)^{2 i}}{(2 i) !} e^{-n^{2} \pi^{2} \tau}<\infty, \quad \forall x \geq 0 .
$$

For $i \geq 0$ let $h_{i}(x):=e^{-\tau \pi^{2} x^{2}}(\pi x)^{2 i}$ and $N_{i}:=$ $\left[\left(\frac{i}{\pi^{2} \tau}\right)^{\frac{1}{2}}\right]$. The map $h_{i}$ is increasing on $\left[0,\left(\frac{i}{\pi^{2} \tau}\right)^{\frac{1}{2}}\right]$ and decreasing on $\left[\left(\frac{i}{\pi^{2} \tau}\right)^{\frac{1}{2}},+\infty\right)$ hence

$$
\begin{aligned}
\sum_{n \geq 0} h_{i}(n) \leq & \int_{0}^{N_{i}} h_{i}(x) d x+h_{i}\left(N_{i}\right) \\
& +h_{i}\left(N_{i}+1\right)+\int_{N_{i+1}}^{\infty} h_{i}(x) d x \\
\leq & 2 h_{i}\left(\left(\frac{i}{\pi^{2} \tau}\right)^{\frac{1}{2}}\right)+\int_{0}^{\infty} h_{i}(x) d x \\
\leq & C \frac{i !}{\tau^{i}}+\int_{0}^{\infty} h_{i}(x) d x
\end{aligned}
$$

we have used Stirling's formula for the last line. On the other hand integrating by parts yields

$$
\begin{aligned}
\int_{0}^{\infty} h_{i}(x) d x & =\frac{2 i-1}{2 \tau} \int_{0}^{\infty} h_{i-1}(x) d x \\
& =\frac{(2 i-1) \cdots 3 \cdot 1}{(2 \tau)^{i}} \int_{0}^{\infty} e^{-\tau \pi^{2} x^{2}} d x \\
& =\frac{(2 i) !}{2^{i} i !(2 \tau)^{i}} \cdot \frac{1}{\pi \sqrt{\tau}} \int_{0}^{\infty} e^{-x^{2}} d x \\
& \leq C \frac{i !}{\tau^{i} \sqrt{\tau}},
\end{aligned}
$$

where we have again used Stirling's formula. Therefore

$$
\left|y_{i}\right| \leq \sqrt{2} \sup _{n \geq 0}\left|c_{n}\right| \sum_{n \geq 0} h_{i}(n) \leq C\left(1+\frac{1}{\sqrt{\tau}}\right) \frac{i !}{\tau^{i}}
$$

Moreover

$$
\sum_{i, n \geq 0} \frac{(n \pi x)^{2 i}}{(2 i) !} e^{-n^{2} \pi^{2} \tau} \leq C\left(1+\frac{1}{\sqrt{\tau}}\right) \sum_{i \geq 0} \underbrace{\frac{i !}{(2 i) !}\left(\frac{x^{2}}{\tau}\right)^{i}}_{=: v_{i}}<\infty
$$

since $\frac{v_{i+1}}{v_{i}} \sim \frac{1}{4 i} \frac{x^{2}}{\tau}$. Lemma 4 is proved.

The following lemma establishes another aspect of the regularizing properties of the heat equation.

Lemma 5 The solution $\theta$ defined by (9) is Gevrey of order 1 in $t$ and $1 / 2$ in $x$ on $[\varepsilon, \tau] \times[0,1]$ for all $\varepsilon \in(0, \tau)$.

Proof of Lemma 5: first notice that for all $\delta>0$

$$
\begin{aligned}
& \left(\delta k^{2} \pi^{2}\right)^{m+\frac{n}{2}} e^{-\frac{3 \delta}{2} k^{2} \pi^{2}} \\
& \quad=\left(\left(\delta k^{2} \pi^{2}\right)^{m} e^{-\delta k^{2} \pi^{2}}\right)\left(\left(\delta k^{2} \pi^{2}\right)^{n} e^{-\delta k^{2} \pi^{2}}\right)^{\frac{1}{2}} \\
& \quad \leq m ! n !^{\frac{1}{2}}
\end{aligned}
$$

by using $x^{p} / p ! \leq e^{x}$ twice. Hence for all $m, n \geq 0$

$$
\begin{aligned}
& \left|\partial_{t}^{m} \partial_{x}^{n} \theta(t, x)\right| \\
& \quad \leq \sqrt{2} \sup _{k \geq 0}\left|c_{k}\right| \sum_{k \geq 0}(k \pi)^{2 m+n} e^{-\varepsilon k^{2} \pi^{2}} \\
& \quad \leq \sqrt{2} \sup _{k \geq 0}\left|c_{k}\right| \sum_{k \geq 0} \frac{\left(\delta k^{2} \pi^{2}\right)^{m+\frac{n}{2}} e^{-\frac{3 \delta}{2} k^{2} \pi^{2}}}{\delta^{m+\frac{n}{2}}} e^{-\left(\varepsilon-\frac{3 \delta}{2}\right) k^{2} \pi^{2}} \\
& \quad \leq\left(\sqrt{2} \sup _{k \geq 0}\left|c_{k}\right| \sum_{k \geq 0} e^{-\left(\varepsilon-\frac{3 \delta}{2}\right) k^{2} \pi^{2}}\right) \frac{m !}{\delta^{m}} \frac{n ! \frac{1}{2}}{\delta^{\frac{n}{2}}} .
\end{aligned}
$$

Picking any $\delta<\frac{2 \varepsilon}{3}$ the last series is obviously convergent, which proves $\theta$ is Gevrey of order 1 in $t$ and $1 / 2$ in $x$ on $[\varepsilon, \tau] \times[0,1]$ for all $\varepsilon \in(0, \tau)$.

Step 2: CONSTRUCtion OF The CONTROL ON $[\tau, T]$

Lemma 6 Let $0<\tau<T$ and $1<s<2$. The function $y:[\tau, T] \rightarrow \mathbb{R}$

$$
y(t):=\phi_{s}\left(\frac{t-\tau}{T-\tau}\right) \sqrt{2} \sum_{n \geq 0} c_{n} e^{-n^{2} \pi^{2} t} .
$$

is Gevrey of order s on $[\tau, T]$ and satisfies for all $i \geq 0$

$$
\begin{aligned}
y^{(i)}(\tau) & =y_{i} \\
y^{(i)}(T) & =0 \\
\left|y^{(i)}(t)\right| & \leq C \sup _{n \geq 0}\left|c_{n}\right| \frac{i !^{s}}{R^{i}}, \quad \forall t \in[\tau, T]
\end{aligned}
$$

for some constants $C, R>0$ depending only on $\tau, T, s$; $\phi_{s}$ is a Gevrey "step function" (see introduction) and the $y_{i}$ 's are as in Lemma 4.

Proof of Lemma 6: $\tilde{y}(t):=\sqrt{2} \sum_{n \geq 0} c_{n} e^{-n^{2} \pi^{2} t}$ is clearly analytic on $(0,+\infty)$ hence Gevrey of order $s$ on $[\tau, T]$, and satisfies for all $i \geq 0$

$$
\tilde{y}^{(i)}(\tau)=\sqrt{2} \sum_{n \geq 0} c_{n} e^{-n^{2} \pi^{2} \tau}\left(-n^{2} \pi^{2}\right)^{i}=y_{i} .
$$


As a consequence $y$ is Gevrey of order $s$ on $[\tau, T]$, and satisfies $y^{(i)}(\tau)=y_{i}$ and $y^{(i)}(T)=0$.

Finally for all $t \in[\tau, T]$

$$
\begin{aligned}
\left|\bar{y}^{(i)}(t)\right| & \leq \sqrt{2} \sup _{n \geq 0}\left|c_{n}\right| \sum_{n \geq 0} e^{-n^{2} \pi^{2} \tau}\left(n^{2} \pi^{2}\right)^{i} \\
& \leq \sqrt{2} \sup _{n \geq 0}\left|c_{n}\right| \frac{i !}{\left(\frac{\tau}{2}\right)^{i}} \sum_{n \geq 0} e^{-n^{2} \pi^{2} \frac{\tau}{2}}
\end{aligned}
$$

where we have used $x^{i} / i ! \leq e^{x}$ for $x=n^{2} \pi^{2} \tau / 2$; (13) then follows from [28, Theorem 19.7]. The proof of Lemma 6 is complete.

The control defined on $[\tau, T]$ by

$$
u(t):=\sum_{i \geq 1} \frac{y^{(i)}(t)}{(2 i-1) !}
$$

steers the system (1)-(3) from $\theta_{\tau}$ at time $\tau$ to 0 at time $T$. Indeed the solution $\theta$ on $[\tau, T]$ is given by (4), and by (11)-(12) satisfies $\theta(\tau, \cdot)=\theta_{\tau}$ and $\theta(T, \cdot)=0$. By Proposition 1 this solution $\theta$ is Gevrey of order $s$ in $t$ and $s / 2$ in $x$ on $[\tau, T] \times[0,1]$; on the other hand by Lemma 5 the solution $\theta$ defined by (9) is Gevrey of order 1 in $t$ and $1 / 2$ in $x$ on $[\varepsilon, \tau] \times[0,1]$ for all $\varepsilon \in(0, \tau)$. Since $s>1$, to prove $\theta$ is Gevrey of order $s$ in $t$ and $s / 2$ in $x$ on $[\varepsilon, T] \times[0,1]$ for all $\varepsilon \in(0, T)$ it is then sufficient to check $\partial_{t}^{k} \theta\left(\tau^{+}, x\right)=\partial_{t}^{k} \theta\left(\tau^{-}, x\right)$ for $k \geq 0$ and $x \in[0,1]$. But by (11) the series (4) and (9) coincide at $t=\tau$, hence so do their (space) derivatives. Therefore

$$
\partial_{t}^{k} \theta\left(\tau^{+}, x\right)=\partial_{x}^{2 k} \theta\left(\tau^{+}, x\right)=\partial_{x}^{2 k} \theta\left(\tau^{-}, x\right)=\partial_{t}^{k} \theta\left(\tau^{-}, x\right) .
$$

As a simple consequence $u=\theta_{x}(t, 1)$ is Gevrey of order $s$ on $[0, T]$, which concludes the proof of Theorem 3 .

\section{The $\mathrm{N}$-dimensional heat equation}

Let $\omega \subset \mathbb{R}^{N-1}, N \geq 2$, be a smooth 1 bounded open set, and $\Omega:=\omega \times(0,1) \subset \mathbb{R}^{N} ; x \in \Omega$ is written $x=\left(x^{\prime}, x_{N}\right)$ with $x^{\prime}=\left(x_{1}, \ldots, x_{N-1}\right) \in \omega$ and $x_{N} \in(0,1)$. We are concerned with the null controllability of the system

$$
\begin{aligned}
\partial_{t} \theta(t, x) & =\Delta \theta(t, x), & & t \in(0, T), x \in \Omega \\
\partial_{\nu} \theta\left(t, x^{\prime}, 1\right) & =u\left(t, x^{\prime}\right), & & t \in(0, T), x^{\prime} \in \omega \\
\partial_{\nu} \theta(t, x) & =0, & & t \in(0, T), x \in \partial \Omega \backslash \omega \times\{1\}(16)
\end{aligned}
$$

$\left(\Delta:=\partial_{x_{1}}^{2}+\cdots+\partial_{x_{N}}^{2}, \nu\right.$ is the outward unit normal vector to $\partial \Omega)$, with initial condition in $L^{2}(\Omega)$

$$
\theta(0, x)=\theta_{0}(x), \quad x \in \Omega .
$$

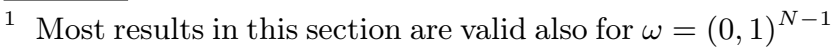

Let $\left(e_{j}\right)_{j \geq 0}$ be an orthonormal basis of $L^{2}(\omega)$ such that each $e_{j}$ is an eigenfunction for the Neumann Laplacian on $\omega$. In other words $e_{j} \in H^{2}(\omega)$ and

$$
\begin{aligned}
-\Delta^{\prime} e_{j}\left(x^{\prime}\right) & =\lambda_{j} e_{j}\left(x^{\prime}\right), & & x^{\prime} \in \omega \\
\frac{\partial e_{j}}{\partial \nu^{\prime}}\left(x^{\prime}\right) & =0, & & x^{\prime} \in \partial \omega,
\end{aligned}
$$

where $\Delta^{\prime}:=\partial_{x_{1}}^{2}+\cdots+\partial_{x_{N-1}}^{2}, \nu^{\prime}$ is the outward unit normal vector to $\partial \omega, 0=\lambda_{0}<\lambda_{1} \leq \lambda_{j} \leq \lambda_{j+1}$, and $e_{0}(x)=|\omega|^{-1 / 2}$. Note that by Weyl's formula, see e.g. [26, Chapter 8][29, Section 15], there are constants $A_{1}, A_{2}>0$ such that

$$
A_{1} j^{\frac{2}{N-1}} \leq \lambda_{j} \leq A_{2} j^{\frac{2}{N-1}}, \quad j \geq 0 .
$$

Now decompose $\theta\left(t, x^{\prime}, 0\right)$ as

$$
\theta\left(t, x^{\prime}, 0\right)=\sum_{j \geq 0} z_{j}(t) e_{j}\left(x^{\prime}\right) .
$$

We claim (14)-(16) is "flat" with $z(t):=\left(z_{j}(t)\right)_{j \geq 0}$ as a flat output, i.e. there is a 1-1 correspondence between arbitrary functions $t \mapsto z(t)$ in a certain space and smooth solutions $\theta$ of (14)-(16). Indeed the $z_{k}$ 's hence $z$ are obviously uniquely defined by a solution $\theta$ of (14) since

$$
\begin{aligned}
\int_{\omega} \theta\left(t, x^{\prime}, 0\right) e_{k}\left(x^{\prime}\right) d x^{\prime} & =\sum_{j \geq 0} z_{j}(t) \int_{\omega} e_{j}\left(x^{\prime}\right) e_{k}\left(x^{\prime}\right) d x^{\prime} \\
& =z_{k}(t) .
\end{aligned}
$$

Conversely, given a sequence $z(t)=\left(z_{j}(t)\right)_{j \geq 0}$ of functions in $C^{\infty}([0, T])$ we seek a formal solution in the form

$$
\theta\left(t, x^{\prime}, x_{N}\right)=\sum_{i \geq 0} \frac{x_{N}^{i}}{i !} a_{i}\left(t, x^{\prime}\right)
$$

where the $a_{i}$ 's are functions yet to be defined. Plugging this formal solution into (14) we find

$$
\sum_{i \geq 0} \frac{x_{N}^{i}}{i !}\left[a_{i+2}\left(t, x^{\prime}\right)-\left(\partial_{t}-\Delta^{\prime}\right) a_{i}\left(t, x^{\prime}\right)\right]=0,
$$

hence $a_{i+2}=\left(\partial_{t}-\Delta^{\prime}\right) a_{i}$ for all $i \geq 0$. Moreover $a_{0}\left(t, x^{\prime}\right)=\theta\left(t, x^{\prime}, 0\right)=\sum_{j \geq 0} z_{j}(t) e_{j}\left(\overline{x^{\prime}}\right)$ by (18) and $a_{1}=0$ by (16). Therefore for all $i \geq 0$,

$$
\begin{aligned}
a_{2 i+1} & =0 \\
a_{2 i} & =\left(\partial_{t}-\Delta^{\prime}\right)^{i} a_{0} \\
& =\sum_{j \geq 0}\left(\partial_{t}-\Delta^{\prime}\right)^{i}\left[z_{j}(t) e_{j}\left(x^{\prime}\right)\right] \\
& =\sum_{j \geq 0} e_{j}\left(x^{\prime}\right)\left(\partial_{t}+\lambda_{j}\right)^{i} z_{j}(t) \\
& =\sum_{j \geq 0} e_{j}\left(x^{\prime}\right) e^{-\lambda_{j} t} y_{j}^{(i)}(t),
\end{aligned}
$$


where we have set $y_{j}(t):=e^{\lambda_{j} t} z_{j}(t)$. Clearly

$$
\begin{aligned}
\theta\left(t, x^{\prime}, x_{N}\right) & =\sum_{j \geq 0} e^{-\lambda_{j} t} e_{j}\left(x^{\prime}\right) \sum_{i \geq 0} y_{j}^{(i)}(t) \frac{x_{N}^{2 i}}{(2 i) !} \\
u\left(t, x^{\prime}\right) & =\sum_{j \geq 0} e^{-\lambda_{j} t} e_{j}\left(x^{\prime}\right) \sum_{i \geq 1} \frac{y_{j}^{(i)}(t)}{(2 i-1) !} .
\end{aligned}
$$

is a formal solution of (14)-(16) uniquely defined by the $y_{j}$ 's hence by the $z_{j}$ 's.

We now give a precise meaning to this formal solution by restricting the $y_{j}$ 's to be Gevrey of order $s \in[1,2)$.

Proposition 7 Let $s \in[1,2), 0<t_{1}<t_{2}<\infty$, and consider the sequence $y=\left(y_{j}\right)_{j>0}$ in $C^{\infty}([0, T])$ satisfying for some constants $M, R>\overline{0}$

$$
\left|y_{j}^{(i)}(t)\right| \leq M \frac{i !^{s}}{R^{i}} \quad \forall i, j \geq 0, \forall t \in\left[t_{1}, t_{2}\right] .
$$

Then the series in (19) is uniformly convergent on $\left[t_{1}, t_{2}\right] \times \bar{\Omega}$ and its sum $\theta$ is Gevrey of order $s$ in $t, 1 / 2$ in $x_{1}, \ldots, x_{N-1}$, and $s / 2$ in $x_{N}$; in particular (14)-(16) and (18) hold for all $(t, x) \in\left[t_{1}, t_{2}\right] \times \bar{\Omega}$. Moreover the formal control $u$ defined by (20) is Gevrey of order $s$ in $t$ and $1 / 2$ in $x_{1}, \ldots, x_{N-1}$ on $\left[t_{1}, t_{2}\right] \times \bar{\omega}$.

\section{PROOF.}

We first prove the formal series

$$
\partial_{t}^{m}\left(\Delta^{\prime}\right)^{l} \partial_{x_{N}}^{n} \theta\left(t, x^{\prime}, x_{N}\right)=\sum_{2 i \geq n} \sum_{j \geq 0} E_{i, j},
$$

where

$$
E_{i, j}:=\frac{x_{N}^{2 i-n}}{(2 i-n) !}\left(-\lambda_{j}\right)^{l} e_{j}\left(x^{\prime}\right) \partial_{t}^{m}\left(e^{-\lambda_{j} t} y_{j}^{(i)}(t)\right)
$$

is uniformly convergent on $\left[t_{1}, t_{2}\right] \times \bar{\Omega}$ with an estimate of the form

$$
\left|\partial_{t}^{m}\left(\Delta^{\prime}\right)^{l} \partial_{x_{N}}^{n} \theta(t, x)\right| \leq C \frac{m !^{s}}{R_{1}^{m}} \frac{(2 l) !^{\frac{1}{2}}}{R_{1}^{2 l}} \frac{n !^{\frac{s}{2}}}{R_{2}^{n}}
$$

for some positive constants $R_{1}, R_{2}, R_{3}$. In what follows $C>0$ denotes a generic constant independent of $m, l, n, t, x$ that may vary from line to line.

Using (21),

$$
\begin{aligned}
\left|E_{i, j}\right| & \leq \frac{\left\|e_{j}\right\|_{L^{\infty}(\omega)}}{(2 i-n) !} \sum_{k=0}^{m}\left(\begin{array}{c}
m \\
k
\end{array}\right)\left|y_{j}^{(i+k)}(t)\right| \lambda_{j}^{l+m-k} e^{-\lambda_{j} t_{1}} \\
& \leq M \frac{m !\left\|e_{j}\right\|_{L^{\infty}(\omega)}^{m}}{(2 i-n) !} \sum_{k=0}^{m} \frac{(i+k) !^{s}}{k ! R^{i+k}} \frac{\lambda_{j}^{l+m-k} e^{-\lambda_{j} t_{1}}}{(m-k) !}
\end{aligned}
$$

Pick any $t_{0} \in\left(0, t_{1}\right)$ such that $\delta:=\frac{t_{1}-t_{0}}{2}<2$, and any integer $\kappa>\frac{N-1}{2}$; by the Sobolev inequalities, see e.g. [9, Section 7.7],

$$
\left\|e_{j}\right\|_{L^{\infty}(\omega)} \leq C\left\|e_{j}\right\|_{H^{\kappa}(\omega)} \leq C \lambda_{j}^{\frac{\kappa}{2}}
$$

hence $\left\|e_{j}\right\|_{L^{\infty}(\omega)} e^{-\delta \lambda_{j}} \leq C$. It follows that

$$
\begin{aligned}
\frac{\lambda_{j}^{l+m-k}\left\|e_{j}\right\|_{L^{\infty}(\omega)} e^{-2 \delta \lambda_{j}}}{(m-k) !} & \leq C \delta^{-(l+m-k)} \frac{(l+m-k) !}{(m-k) !} \\
& \leq C\left(\frac{2}{\delta}\right)^{l+m-k} l !
\end{aligned}
$$

where we have used $x^{p} e^{-x} \leq p !$ and $(p+q) ! \leq 2^{p+q} p ! q$ ! Thus since $s \geq 1$

$$
\begin{aligned}
\left|E_{i, j}\right| & \leq C e^{-\lambda_{j} t_{0}}\left(\frac{2}{\delta}\right)^{l} l ! \frac{m !}{(2 i-n) !} \sum_{k=0}^{m} \frac{2^{m-k+s(i+k)}}{\delta^{m-k} k !} \frac{i !^{s} k !^{s}}{R^{i+k}} \\
& \leq C e^{-\lambda_{j} t_{0}}\left(\frac{2}{\delta}\right)^{l} l ! \frac{i !^{s}\left(\frac{2^{s}}{R}\right)^{i}}{(2 i-n) !}\left(\frac{2}{\delta}\right)^{m} m !^{s} \sum_{k=0}^{m}\left(\frac{2^{s}}{R}\right)^{k} .
\end{aligned}
$$

Now by Stirling's formula

$$
l !\left(\frac{2}{\delta}\right)^{l} \sim(2 l) !^{\frac{1}{2}} \frac{(\pi l)^{\frac{1}{4}}}{\delta^{l}} \leq C \frac{(2 l) !^{\frac{1}{2}}}{R_{2}^{2 l}}
$$

where $R_{2}<\sqrt{\delta}$. Likewise using also $(p+q) ! \leq 2^{p+q} p ! q$ !

$$
\begin{aligned}
\frac{i !^{s}\left(\frac{2^{s}}{R}\right)^{i}}{(2 i-n) !} & \sim\left(\frac{2^{s}}{R}\right)^{i} \frac{(2 i) !^{\frac{s}{2}}}{(2 i-n) !^{\frac{s}{2}}} \frac{(\pi i)^{\frac{s}{4}}}{(2 i-n) !^{1-\frac{s}{2}} 2^{i s}} \\
& \leq\left(\frac{2^{s}}{R}\right)^{i}(\pi i)^{\frac{s}{4}} \frac{n !^{\frac{s}{2}}}{(2 i-n) !^{1-\frac{s}{2}}} \\
& \leq\left(\frac{2^{s}}{R}\right)^{i}\left(\frac{n \pi}{2}\right)^{\frac{s}{4}}\left(1+\left(\frac{2 i-n}{n}\right)^{\frac{s}{4}}\right) \frac{n !^{\frac{s}{2}}}{(2 i-n) !^{1-\frac{s}{2}}} \\
& \leq C \frac{n !^{\frac{s}{2}}}{R_{2}^{n}} \underbrace{\frac{1+\left(\frac{2 i-n}{n}\right)^{\frac{s}{4}}}{(2 i-n) !^{1-\frac{s}{2}}} \sqrt{\frac{2^{s}}{R}} 2 i-n}_{A_{2 i-n}},
\end{aligned}
$$

where $R_{3}<\sqrt{\frac{R}{2^{s}}}$ and $\sum_{2 i \geq n} A_{2 i-n}<\infty$. Finally notice

$$
\left(\frac{2}{\delta}\right)^{m} \sum_{k=0}^{m}\left(\frac{2^{s}}{R}\right)^{k} \leq C \frac{1}{R_{1}^{m}}
$$

if we set

$$
R_{1}:= \begin{cases}\frac{\delta R}{2^{s+1}} & \text { if } R<2^{s} \\ \frac{\delta}{4} & \text { if } R=2^{s} \\ \frac{\delta}{2} & \text { if } R>2^{s}\end{cases}
$$


Collecting the three previous estimates yields

$$
\sum_{2 i \geq n} \sum_{j \geq 0} E_{i, j} \leq C \frac{m !^{s}}{R_{1}^{m}} \frac{(2 l) !^{\frac{1}{2}}}{R_{2}^{2 l}} \frac{n !^{\frac{s}{2}}}{R_{3}^{n}} \sum_{j \geq 0} e^{-\lambda_{j} t_{0}} \sum_{2 i \geq n} A_{2 i-n} .
$$

The second series in the r.h.s. is convergent and so is the first one by (17). Thus (22) is proved, which shows $\partial_{t}^{m}\left(\Delta^{\prime}\right)^{l} \partial_{x_{N}}^{n} \theta \in C\left(\left[t_{1}, t_{2}\right] \times \bar{\Omega}\right)$ for all $m, l, n \geq 0$, hence $\theta \in C^{\infty}\left(\left[t_{1}, t_{2}\right] \times \bar{\Omega}\right)$ by the Sobolev imbedding theorem.

To complete the proof of the proposition we must extend (22) to every derivative $\partial_{t}^{m} \partial_{x^{\prime}}^{\alpha^{\prime}} \partial_{x_{N}}^{n} \theta$ where $\alpha^{\prime}=$ $\left(\alpha_{1}, \ldots, \alpha_{N-1}\right) \in \mathbb{N}^{N-1}$ and $\partial_{x^{\prime}}^{\alpha^{\prime}}=\partial_{x_{1}}^{\alpha_{1}} \ldots \partial_{x_{N-1}}^{\alpha_{N-1}}$. Pick any integer $p \in(N-1, \infty)$. By the Sobolev inequalities

$$
\|v\|_{L^{\infty}(\omega)} \leq C\|v\|_{W^{1, p}(\omega)} .
$$

Repeated applications of the classical elliptic estimate

$$
\|v\|_{W^{2, p}(\omega)} \leq C\left(\|\Delta v\|_{L^{p}(\omega)}+\|v\|_{L^{p}(\omega)}\right)
$$

(where the constant $C$ depends on $p$ ) yield for all $l \in \mathbb{N}^{\star}$

$$
\|v\|_{W^{2 l, p}(\omega)} \leq C\left(\|v\|_{L^{p}(\omega)}+\sum_{k=1}^{l}\left\|\Delta^{k} v\right\|_{L^{p}(\omega)}\right) .
$$

As a consequence and using (24),

$$
\|v\|_{W^{2 l, p}(\omega)} \leq C\left(\|v\|_{L^{\infty}(\omega)}+\sum_{k=1}^{l}\left\|\Delta^{k} v\right\|_{L^{\infty}(\omega)}\right) .
$$

Pick any $l \in \mathbb{N}^{\star}$ and any $\alpha^{\prime} \in \mathbb{N}^{N-1}$ with $\left|\alpha^{\prime}\right|:=\alpha_{1}+$ $\cdots+\alpha_{N-1} \in\{2 l-2,2 l-1\}$. Assume e.g. that $\left|\alpha^{\prime}\right|=$ $2 l-1$, the computations being similar when $\left|\alpha^{\prime}\right|=2 l-2$. Then with (25) and (22),

$$
\begin{aligned}
\left|\partial_{t}^{m} \partial_{x^{\prime}}^{\alpha^{\prime}} \partial_{x_{N}}^{n} \theta(t, x)\right| & \leq\left\|\partial_{t}^{m} \partial_{x_{N}}^{n} \theta\left(t, \cdot, x_{N}\right)\right\|_{W^{2 l-1, \infty}(\omega)} \\
& \leq C \frac{m !^{s}}{R_{1}^{m}} \sum_{k=0}^{l} \frac{(2 k) !^{\frac{1}{2}}}{R_{2}^{2 k}} \frac{n !^{\frac{s}{2}}}{R_{3}^{n}} \\
& \leq C \frac{m !^{s}}{R_{1}^{m}}(l+1) \frac{(2 l) !^{\frac{1}{2}}}{R_{2}^{2 l}} \frac{n !^{\frac{s}{2}}}{R_{3}^{n}}
\end{aligned}
$$

Pick $R_{2}^{\prime} \in\left(0, R_{2}\right)$. Then $(l+1) \sqrt{2 l} \leq C{\frac{R_{2}}{R_{2}^{\prime}}}^{2 l}$ hence

$$
(l+1) \frac{(2 l) !^{\frac{1}{2}}}{R_{2}^{2 l}} \leq C \frac{(2 l-1) !^{\frac{1}{2}}}{\left(R_{2}^{\prime}\right)^{2 l}} \leq C \frac{\alpha_{1} !^{\frac{1}{2}} \cdots \alpha_{N-1} !^{\frac{1}{2}}}{\left(\frac{R_{2}^{\prime}}{\sqrt{N-1}}\right)^{\alpha_{1}+\cdots+\alpha_{N-1}}},
$$

where we have used $(2 l-1) ! \leq(N-1)^{2 l-1} \alpha_{1} ! \cdots \alpha_{N-1} !$. Finally, setting $R_{2}^{\prime \prime}=\frac{R_{2}^{\prime}}{\sqrt{N-1}}$,

$$
\left|\partial_{t}^{m} \partial_{x^{\prime}}^{\alpha^{\prime}} \partial_{x_{N}}^{n} \theta(t, x)\right| \leq C \frac{m !^{s}}{R_{1}^{m}} \frac{\alpha_{1} !^{\frac{1}{2}} \cdots \alpha_{N-1} !^{\frac{1}{2}}}{\left(R_{2}^{\prime \prime}\right)^{\alpha_{1}+\cdots+\alpha_{N-1}}} \frac{n !^{\frac{s}{2}}}{R_{3}^{n}},
$$

which completes the proof of Proposition 7.

With Proposition 7 at hand, we can derive a constructive null controllability result. The approach is similar to and builds on the proof of Theorem 3 .

Theorem 8 Let $\theta_{0} \in L^{2}(\Omega), T>0, \tau \in(0, T)$ and $s \in$ $(1,2)$. Then there exists a sequence $\left(y_{j}\right)_{j \geq 0}$ of functions in $C^{\infty}([\tau, T])$ which are Gevrey of order $s$ on $[\tau, T]$ and such that the control

$u\left(t, x^{\prime}\right)=: \begin{cases}0 & \text { if } 0 \leq t \leq \tau \\ \sum_{i \geq 1, j \geq 0} e^{-\lambda_{j} t} \frac{y_{j}^{(i)}(t)}{(2 i-1) !} e_{j}\left(x^{\prime}\right) & \text { if } \tau<t \leq T .\end{cases}$

steers the system (14)-(16) from the initial state $\theta(0,)=$. $\theta_{0}$ at time 0 to the final state $\theta(T,)=$.0 at time $T$.

Moreover $u$ is Gevrey of order $s$ in $t$ and $1 / 2$ in $x_{1}, \ldots, x_{N-1}$ on $[0, T] \times \bar{\omega}$; the solution $\theta$ of $(14)-(16)$ is Gevrey of order $s$ in $t, 1 / 2$ in $x_{1}, \ldots, x_{N-1}$, and $s / 2$ in $x_{N}$ on $[\varepsilon, T] \times \bar{\Omega}$ for all $\varepsilon \in(0, T)$.

\section{PROOF.}

Step 1: Free Evolution Let $u\left(t, x^{\prime}\right)=0$ for $\left(t, x^{\prime}\right) \in$ $[0, \tau] \times \omega$. Decompose $\theta_{0}$ as the Fourier series of cosines

$$
\theta_{0}\left(x^{\prime}, x_{N}\right)=\sum_{j, n \geq 0} c_{j, n} e_{j}\left(x^{\prime}\right) \sqrt{2} \cos \left(n \pi x_{N}\right),
$$

with convergence in $L^{2}(\Omega)$ and $\sum_{j, n \geq 0}\left|c_{j, n}\right|^{2}<\infty$. The solution $\theta$ of (14)-(16) starting from $\theta_{0}$ then reads

$\theta\left(t, x^{\prime}, x_{N}\right)=\sum_{j, n \geq 0} c_{j, n} e^{-\left(\lambda_{j}+n^{2} \pi^{2}\right) t} e_{j}\left(x^{\prime}\right) \sqrt{2} \cos \left(n \pi x_{N}\right)$.

In particular

$$
\theta_{\tau}(t, x):=\theta\left(\tau, x^{\prime}, x_{N}\right)=\sum_{j \geq 0} e^{-\lambda_{j} \tau} e_{j}\left(x^{\prime}\right) \theta_{\tau, j}\left(x_{N}\right),
$$

where by Lemma 4

$$
\begin{aligned}
\theta_{\tau, j}\left(x_{N}\right) & =\sum_{n \geq 0} c_{j, n} e^{-n^{2} \pi^{2} \tau} \sqrt{2} \cos \left(n \pi x_{N}\right) \\
& =\sum_{i \geq 0} \frac{x_{N}^{2 i}}{(2 i) !} \underbrace{\left(\sqrt{2} \sum_{n \geq 0} c_{j, n} e^{-n^{2} \pi^{2} \tau}\left(-n^{2} \pi^{2}\right)^{i}\right)}_{=: y_{j, i}}
\end{aligned}
$$

with $\left|y_{j, i}\right| \leq C\left(1+\frac{1}{\sqrt{\tau}}\right) \frac{i !}{\tau^{i}}$. 
SteP 2: CONSTRUCTION OF THE CONTROL ON $[\tau, T]$ By Lemma 6 the functions $y_{j}, j \geq 0$, defined on $[\tau, T]$ by

$$
y_{j}(t):=\phi_{s}\left(\frac{t-\tau}{T-\tau}\right) \sqrt{2} \sum_{n \geq 0} c_{j, n} e^{-n^{2} \pi^{2} t}
$$

are Gevrey of order $s$ on $[\tau, T]$, and satisfy for all $i \geq 0$

$$
\begin{aligned}
y_{j}^{(i)}(\tau) & =y_{j, i} \\
y_{j}^{(i)}(T) & =0 \\
\left|y_{j}^{(i)}(t)\right| & \leq C \sup _{j, n \geq 0}\left|c_{j, n}\right| \frac{i !^{s}}{R^{i}}, \quad \forall t \in[\tau, T]
\end{aligned}
$$

for some constants $C, R>0$ depending only on $\tau, T, s$.

The control defined on $[\tau, T]$ by (20) then steers the system (14)-(16) from $\theta_{\tau}$ at time $\tau$ to 0 at time $T$. Indeed the solution $\theta$ on $[\tau, T]$ is given by (19), and by (29)(30) satisfies $\theta(\tau, \cdot)=\theta_{\tau}$ and $\theta(T, \cdot)=0$. By Proposition 7 this solution $\theta$ is Gevrey of order $s$ in $t, 1 / 2$ in $x_{1}, \ldots, x_{N-1}$, and $s / 2$ in $x_{N}$; on the other hand by an easy extension of Lemma 5 and arguments similar to those in the proof of Proposition 7 the solution $\theta$ defined by (27) is Gevrey of order 1 in $t$ and $1 / 2$ in $x_{1}, \ldots, x_{N}$ on $[\varepsilon, \tau] \times \bar{\Omega}$ for all $\varepsilon \in(0, \tau)$. Since $s>1$, to prove $\theta$ is Gevrey of order $s$ in $t, 1 / 2$ in $x_{1}, \ldots, x_{N-1}$, and $s / 2$ in $x_{N}$ on $[\varepsilon, T] \times \bar{\Omega}$ for all $\varepsilon \in(0, T)$ it is then sufficient to check $\partial_{t}^{k} \theta\left(\tau^{+}, x\right)=\partial_{t}^{k} \theta\left(\tau^{-}, x\right)$ for $k \geq 0$ and $x \in \bar{\Omega}$. But by (29) the series (19) and (27) coincide at $t=\tau$, hence so do their (space) derivatives. Therefore

$\partial_{t}^{k} \theta\left(\tau^{+}, x\right)=\Delta_{x}^{2 k} \theta\left(\tau^{+}, x\right)=\Delta_{x}^{2 k} \theta\left(\tau^{-}, x\right)=\partial_{t}^{k} \theta\left(\tau^{-}, x\right)$.

As a simple consequence $u\left(t, x^{\prime}\right)=\partial_{x_{N}} \theta\left(t, x^{\prime}, 1\right)$ is Gevrey of order $s$ in $t$ and $1 / 2$ in $x_{1}, \ldots, x_{N-1}$ on $[0, T] \times \bar{\omega}$, which completes the proof of Theorem 8 .

\section{Numerical approximations}

We now investigate numerical approximations of the control problem (14)-(16). We assume $N \geq 2$, the case $N=1$ being similar and simpler. From section 4 we know the control $u$ achieving the exact control to zero is explicitly given by Theorem 8 , with the functions $y_{j}$ and the coefficients $c_{j, n}$ defined by (28)-(26); the corresponding solution $\theta$ is given by (27) for $t \leq \tau$ and by (19) for $t \geq \tau$. The aim of this section is to show that approximating the various series by their truncated sums at given $\bar{\imath}, \bar{\jmath}, \bar{n} \in \mathbb{N}$ provides very good approximations, and to give explicit error estimates.

We begin with the free evolution and consider

$$
\bar{\theta}\left(t, x^{\prime}, x_{N}\right):=\sqrt{2} \sum_{\substack{0 \leq j \leq \bar{j} \\ 0 \leq n \leq \bar{n}}} c_{j, n} e^{-\left(\lambda_{j}+n^{2} \pi^{2}\right) t} e_{j}\left(x^{\prime}\right) \cos \left(n \pi x_{N}\right)
$$

instead of the true solution (27). Clearly for $0 \leq t \leq \tau$

$$
\|\theta(t)-\bar{\theta}(t)\|_{L^{2}(\Omega)}^{2}=\sum_{(j, n) \notin[0, \bar{j}] \times[0, \bar{n}]} e^{-2\left(\lambda_{j}+n^{2} \pi^{2}\right) t}\left|c_{j, n}\right|^{2}
$$

hence $\bar{\theta} \rightarrow \theta$ in $C\left([0, \tau] ; L^{2}(\Omega)\right)$ as $\bar{\jmath}, \bar{n} \rightarrow \infty$. Moreover for $\tau^{\prime} \in(0, \tau)$ and $r>0, \bar{\theta} \rightarrow \theta$ in $C\left(\left[\tau^{\prime}, \tau\right] ; H^{r}(\Omega)\right)$ as $\bar{\jmath}, \bar{n} \rightarrow \infty$.

The main result of this section concerns the convergence for $t \in[\tau, T]$ of the truncated sums

$$
\begin{aligned}
\bar{u}\left(t, x^{\prime}\right) & :=\sum_{0 \leq j \leq \bar{j}} e^{-\lambda_{j} t} e_{j}\left(x^{\prime}\right) \sum_{1 \leq i \leq \bar{\imath}} \frac{{\overline{y_{j}}}^{(i)}(t)}{(2 i-1) !} \\
\bar{\theta}\left(t, x^{\prime}, x_{N}\right) & :=\sum_{0 \leq j \leq \bar{j}} e^{-\lambda_{j} t} e_{j}\left(x^{\prime}\right) \sum_{0 \leq i \leq \bar{\imath}}{\overline{y_{j}}}^{(i)}(t) \frac{x_{N}^{2 i}}{(2 i) !} \\
\overline{y_{j}}(t) & :=\phi_{s}\left(\frac{t-\tau}{T-\tau}\right) \sqrt{2} \sum_{0 \leq n \leq \bar{n}} c_{j, n} e^{-n^{2} \pi^{2} t} .
\end{aligned}
$$

Let also $\hat{u}$ be the control defined by 0 for $t \in[0, \tau]$ and by $\bar{u}$ for $t \in(\tau, T]$, and $\hat{\theta}$ be the solution of (14)-(16) when $u$ is replaced by $\hat{u}$ (still starting from $\theta_{0}$ at time $0) ; \hat{\theta}$ is thus the solution of the "true" control problem when using the approximated control $\hat{u}$ instead of the "ideal" control $u$. Notice $\hat{\theta}$ and $\theta$ agree on $[0, \tau]$, but $\hat{\theta}$ and $\bar{\theta}$ differ on $(\tau, T]$ (i.e. the solution produced by the truncated control is not the truncated solution).

Theorem 9 Let $\theta_{0} \in L^{2}(\Omega), T>0, \tau \in(0, T), s \in$ $(1,2)$ and $N \geq 2$. Then there are constants $C_{1}, C_{2}, C_{3}, C_{4}$ such that for all $t \in[\tau, T]$

$$
\|\theta(t)-\bar{\theta}(t)\|_{L^{\infty}(\Omega)} \leq C_{1} f(\bar{\imath}, \bar{\jmath}, \bar{n})\left\|\theta_{0}\right\|_{L^{2}(\Omega)},
$$

where

$$
f(\bar{\imath}, \bar{\jmath}, \bar{n}):=e^{-C_{2} \bar{\jmath}^{\frac{2}{N-1}}}+e^{-C_{3} \bar{\imath} \ln \bar{\imath}}+e^{-C_{4} \bar{n}^{2}} .
$$

In (32)-(33) one may choose $C_{2}<A_{1} \tau$ (see (17)), $C_{3}<$ $2-s$, and $C_{4}<\pi^{2} \tau$, while $C_{1}=C_{1}\left(N, \omega, \tau, s, C_{2}, C_{3}, C_{4}\right)$.

PROOF. For $\left(t, x^{\prime}, x_{N}\right) \in[\tau, T] \times \omega \times[0,1]$

$$
\left|\theta\left(t, x^{\prime}, x_{N}\right)-\bar{\theta}\left(t, x^{\prime}, x_{N}\right)\right| \leq \Delta_{1}+\Delta_{2}+\Delta_{3},
$$


where

$$
\begin{aligned}
\Delta_{1} & :=\sum_{j>\bar{\jmath}} e^{-\lambda_{j} t}\left|e_{j}\left(x^{\prime}\right)\right| \sum_{i \geq 0} \frac{\left|y_{j}^{(i)}(t)\right|}{(2 i) !} \\
\Delta_{2} & :=\sum_{0 \leq j \leq \bar{\jmath}} e^{-\lambda_{j} t}\left|e_{j}\left(x^{\prime}\right)\right| \sum_{i>\bar{\imath}} \frac{\left|y_{j}^{(i)}(t)\right|}{(2 i) !} \\
\Delta_{3} & :=\sum_{0 \leq j \leq \bar{\jmath}} e^{-\lambda_{j} t}\left|e_{j}\left(x^{\prime}\right)\right| \sum_{0 \leq i \leq \bar{\imath}} \frac{\left|\partial_{t}^{i}\left[\phi(t) \sum_{n>\bar{n}} c_{j, n} e^{-n^{2} \pi^{2} t}\right]\right|}{(2 i) !}
\end{aligned}
$$

and $\phi(t):=\sqrt{2} \phi_{s}\left(\frac{t-\tau}{T-\tau}\right)$.

We first bound $\Delta_{1}$. On the one hand by (31) and $s<2$

$$
\begin{aligned}
\sum_{i \geq 0} \frac{\left|y_{j}^{(i)}(t)\right|}{(2 i) !} & \leq C \sup _{j, n \geq 0}\left|c_{j, n}\right| \sum_{i \geq 0} \frac{(i !)^{s}}{(2 i) ! R^{i}} \\
& \leq C \sup _{j, n \geq 0}\left|c_{j, n}\right|
\end{aligned}
$$

for some constant $C=C(\tau, T, s)$. On the other hand using (23), (17) and $x^{p} e^{-x} \leq p$ ! and taking $\rho \in(0,1)$

$$
\begin{aligned}
e^{-\lambda_{j} \tau}\left\|e_{j}\right\|_{L^{\infty}(\omega)} & \leq C e^{-\lambda_{j} \tau} \lambda_{j}^{\frac{\kappa}{2}} \\
& \leq C e^{-\lambda_{j} \rho \tau} \\
& \leq C e^{-A_{1} \rho \tau j \frac{2}{N-1}}
\end{aligned}
$$

where the constant $C$ eventually depends on $\tau, N$. But

$$
\begin{aligned}
\sum_{j>\bar{\jmath}} e^{-K j^{r}} & \leq \int_{\bar{\jmath}}^{\infty} e^{-K x^{r}} d x \\
& =\frac{1}{r} \int_{\bar{\jmath}^{r}}^{\infty} e^{-K y} y^{\frac{1}{r}-1} d y \\
& \leq \begin{cases}\frac{\bar{\jmath}^{1-r}}{r K} e^{-K \bar{j}^{r}} & \text { if } r \geq 1 \\
K^{\prime} e^{-K^{\prime \prime} \bar{\jmath}^{r}} & \text { if } r<1,\end{cases}
\end{aligned}
$$

where $r, K, K^{\prime}>0$ and $K^{\prime \prime} \in(0, K)$. Picking $C_{2}<A_{1} \tau$ then yields

$$
\sum_{j>\bar{\jmath}} e^{-\lambda_{j} \tau}\left\|e_{j}\right\|_{L^{\infty}(\omega)} \leq C e^{-C_{2} \bar{\jmath} \frac{2}{N^{-1}}},
$$

where $C=C\left(\tau, N, C_{2}\right)$. We conclude by (35) and (37)

$$
\begin{aligned}
\Delta_{1} & \leq \sum_{j>\bar{\jmath}} e^{-\lambda_{j} \tau}\left\|e_{j}\right\|_{L^{\infty}(\omega)} \sum_{i \geq 0} \frac{\left|y_{j}^{(i)}(t)\right|}{(2 i) !} \\
& \leq C e^{-C_{2} \bar{\jmath}^{\frac{2}{N-1}}} \sup _{j, n \geq 0}\left|c_{j, n}\right|,
\end{aligned}
$$

where $C=C\left(\tau, T, N, C_{2}, s\right)$.
We next bound $\Delta_{2}$. By (31) and using Stirling's formula for $i$ ! and $(2 i)$ !

$$
\begin{aligned}
\sum_{i \geq \bar{\imath}} \frac{\left|y_{j}^{(i)}(t)\right|}{(2 i) !} & \leq C \sup _{j, n \geq 0}\left|c_{j, n}\right| \sum_{i \geq \bar{\imath}} \frac{(i !)^{s}}{(2 i) ! R^{i}} \\
& \leq C \sup _{j, n \geq 0}\left|c_{j, n}\right| \sum_{i \geq \bar{\imath}} \frac{i^{\frac{s-1}{2}}}{\left(\frac{i}{e}\right)^{(2-s) i}(4 R)^{i}} \\
& \leq C \sup _{j, n \geq 0}\left|c_{j, n}\right| \sum_{i \geq \bar{\imath}} \frac{i^{\frac{s-1}{2}} e^{-(2-s) i(\ln i-1)}}{(4 R)^{i}}
\end{aligned}
$$

for some constant $C=C(\tau, T, s)$. Pick $C_{3}<2-s$ and $\sigma \in\left(C_{3}, 2-s\right)$; then

$$
\frac{i^{\frac{s-1}{2}} e^{(2-s)(1-\ln i) i}}{(4 R)^{i}} \leq C e^{-\sigma i(\ln i-1)}
$$

where $C=C(s, \sigma, R)$, and

$$
\begin{aligned}
\sum_{i>\bar{\imath}} e^{-\sigma i(\ln i-1)} & \leq \int_{\bar{\imath}}^{\infty} e^{-\sigma x(\ln x-1)} d x \\
& \leq C \int_{\bar{\imath}(\ln \bar{\imath}-1)}^{\infty} e^{-\sigma y} d y \\
& \leq C e^{-C_{3} \bar{\imath} \ln \bar{\imath}}
\end{aligned}
$$

We conclude by (36)

$$
\begin{aligned}
\Delta_{2} & \leq \sum_{0 \leq j \leq \bar{j}} e^{-\lambda_{j} \tau}\left\|e_{j}\right\|_{L^{\infty}(\omega)} \sum_{i \geq \bar{\imath}} \frac{\left|y_{j}^{(i)}(t)\right|}{(2 i) !} \\
& \leq C \sup _{j, n \geq 0}\left|c_{j, n}\right| e^{-C_{3} \bar{\imath} \ln \bar{\imath}} \sum_{j \geq 0} e^{-A_{1} \rho \tau j \frac{2}{N-1}} \\
& \leq C \sup _{j, n \geq 0}\left|c_{j, n}\right| e^{-C_{3} \bar{\imath} \ln \bar{\imath}}
\end{aligned}
$$

where $C=C\left(\tau, T, N, s, C_{3}\right)$, since the last series clearly converges.

We finally bound $\Delta_{3}$. Pick $\rho \in(0,1)$ and notice first

$$
\sum_{n>\bar{n}} e^{-n^{2} \pi^{2} \rho \tau} \leq e^{-\bar{n}^{2} \pi^{2} \rho \tau} \sum_{p \geq 1} e^{-p^{2} \pi^{2} \rho \tau} \leq C e^{-\bar{n}^{2} \pi^{2} \rho \tau},
$$


the series being clearly convergent. Then for $t \geq \tau$

$$
\begin{aligned}
\left|\partial_{t}^{i} \sum_{n>\bar{n}} c_{j, n} e^{-n^{2} \pi^{2} t}\right| & =\left|\sum_{n>\bar{n}} c_{j, n}\left(-n^{2} \pi^{2}\right)^{i} e^{-n^{2} \pi^{2} t}\right| \\
& \leq \sup _{j, n \geq 0}\left|c_{j, n}\right| \sum_{n>\bar{n}}\left(n^{2} \pi^{2}\right)^{i} e^{-n^{2} \pi^{2} \tau} \\
& \leq i ! \frac{\sup _{j, n \geq 0}\left|c_{j, n}\right|}{((1-\rho) \tau)^{i}} \sum_{n>\bar{n}} e^{-n^{2} \pi^{2} \rho \tau} \\
& \leq C \sup _{j, n \geq 0}\left|c_{j, n}\right| \frac{i ! e^{-\bar{n}^{2} \pi^{2} \rho \tau}}{((1-\rho) \tau)^{i}}
\end{aligned}
$$

where we have used the estimate $x^{i} / i$ ! $\leq e^{x}$ for $x=$ $n^{2} \pi^{2}(1-\rho) \tau$. In other words $t \mapsto \sum_{n>\bar{n}} c_{j, n} e^{-n^{2} \pi^{2} t}$ is Gevrey of order 1 hence $s$ on $[\tau, T]$. Since the product of functions Gevrey of order $s$ is also Gevrey of order $s$

$$
\left|\partial_{t}^{i}\left[\phi(t) \sum_{n>\bar{n}} c_{j, n} e^{-n^{2} \pi^{2} t}\right]\right| \leq C e^{-\bar{n}^{2} \pi^{2} \rho \tau} \sup _{j, n \geq 0}\left|c_{j, n}\right| \frac{i !^{s}}{R^{i}}
$$

for some $C, R$ depending on $\tau, T, \rho, s$. We conclude by $(36)$

$$
\begin{aligned}
\Delta_{3} & \leq \sum_{0 \leq j \leq \bar{j}} e^{-\lambda_{j} \tau}\left\|e_{j}\right\|_{L^{\infty}(\omega)} \sum_{0 \leq i \leq \bar{\imath}} C \sup _{j, n \geq 0}\left|c_{j, n}\right| \frac{i !^{s} e^{-C_{4} \bar{n}^{2}}}{(2 i) ! R^{i}} \\
& \leq C e^{-C_{4} \bar{n}^{2}} \sup _{j, n \geq 0}\left|c_{j, n}\right| \sum_{j \geq 0} e^{-A_{1} \rho \tau j \frac{2}{N-1}} \sum_{i \geq 0} \frac{i !^{s}}{(2 i) ! R^{i}} \\
& \leq C e^{-C_{4} \bar{n}^{2}} \sup _{j, n \geq 0}\left|c_{j, n}\right|
\end{aligned}
$$

where $C=C(\tau, T, \rho, s)$, since the last two series clearly converge; we have set $C_{4}:=\pi^{2} \rho \tau$.

Collecting (34), (38), (39) and (40) then yields (32).

Corollary 10 Using the notations of Theorem 9 and $q \in \mathbb{N}^{\star}$, there are constants $C_{1}^{\prime}, C_{1}^{\prime \prime}$ depending on $N, \omega, \tau, s, C_{2}, C_{3}, C_{4}, q$ such that

$$
\begin{aligned}
\|\theta-\bar{\theta}\|_{W^{q, \infty}((\tau, T) \times \Omega)} & \leq C_{1}^{\prime} f(\bar{\imath}, \bar{\jmath}, \bar{n})\left\|\theta_{0}\right\|_{L^{2}(\Omega)} \\
\|u-\bar{u}\|_{W^{q-1, \infty}((\tau, T) \times \Omega)} & \leq C_{1}^{\prime \prime} f(\bar{\imath}, \bar{\jmath}, \bar{n})\left\|\theta_{0}\right\|_{L^{2}(\Omega)}
\end{aligned}
$$

PROOF. The proof of (41) runs along the same lines as the proof of Theorem 9. Indeed any additional factor in the $\Delta_{i}$ 's produced by a derivation in $t, x^{\prime}$ or $x_{N}$ is absorbed by $e^{-\lambda_{j} \tau}$ or $i !^{s-2}$. On the other hand (42) follows at once from (41) and the fact that $\bar{u}=\partial_{x_{N}} \bar{\theta}\left(t, x^{\prime}, 1\right)$.

Corollary 11 Using the notations of Theorem 9, there is a constant $C_{1}^{\prime \prime \prime}=C_{1}^{\prime \prime \prime}\left(N, \omega, \tau, s, C_{2}, C_{3}, C_{4}\right)$ such that

$$
\|\theta-\hat{\theta}\|_{L^{\infty}((0, T) \times \Omega)} \leq C_{1}^{\prime \prime \prime} f(\bar{\imath}, \bar{\jmath}, \bar{n})\left\|\theta_{0}\right\|_{L^{2}(\Omega)}
$$

PROOF. Let

$$
\tilde{\theta}:=\theta-\hat{\theta}-\frac{x_{N}^{2}}{2}\left(u\left(t, x^{\prime}\right)-\bar{u}\left(t, x^{\prime}\right)\right)
$$

$\tilde{\theta}$ is the solution of the initial-boundary-value problem

$$
\begin{aligned}
\partial_{t} \theta(t, x) & -\Delta \tilde{\theta}(t, x) & & \\
& =\left(\Delta-\partial_{t}\right)\left[\frac{x_{N}^{2}}{2}(u-\bar{u})\right], & & t \in(0, T), x \in \Omega \\
\partial_{\nu} \tilde{\theta}(t, x) & =0, & & t \in(0, T), x \in \partial \Omega \\
\tilde{\theta}(0, x) & =0, & & x \in \Omega .
\end{aligned}
$$

Then (43) follows from (42) with $q=3$ and classical semigroup estimates in $C(\bar{\Omega})$.

\section{Numerical experiments}

We illustrate the approach on two numerical examples, in dimension 1 and 2 .

\subsection{Example in dimension 1}

We have taken $T=0.35, \tau=0.05, s=1.65$. The initial condition $\theta_{0}$ is a step function with $\theta_{0}(x)=-0.75$ on $[0,1 / 2)$ and $\theta_{0}(x)=1.25$ on $(1 / 2,1)$, with nonzero Fourier coefficients $c_{0}=\frac{\sqrt{2}}{8}$ and $c_{2 p+1}=\frac{(-1)^{p+1}}{2 p+1} \frac{2 \sqrt{2}}{\pi}$ for $p \geq 0$; notice $\theta_{0}$ is not continuous and that its Fourier coefficients decay fairly slowly. The series for the control $u$ and the function $y$ of Theorem 3 have been truncated at a "large enough" order for a good accuracy, namely $\bar{\imath}=35$ and $\bar{n}=25$. For the Gevrey "step function" we have used

$$
\phi_{s}(t):= \begin{cases}1 & \text { if } t \leq 0 \\ 0 & \text { if } t \geq 1 \\ 1-\frac{\int_{0}^{t} \varphi_{s}(\rho) d \rho}{\int_{0}^{1} \varphi_{s}(\rho) d \rho} & \text { if } t \in(0,1),\end{cases}
$$

where $k=(s-1)^{-1}$ and $\varphi_{s}$ is the "bump function"

$$
\varphi_{s}(t):= \begin{cases}0 & \text { if } t \notin(0,1) \\ \exp \left(\frac{-1}{2 t^{k}(1-t)^{k}}\right) & \text { if } t \in(0,1) .\end{cases}
$$

This "step function" was preferred to the one in section 2 because its derivatives are easier to compute formally.

Fig. 1 shows the (truncated) control $\hat{u}$ given by Theorem 3 , and Fig. 2 the resulting simulated temperature $\hat{\theta}$ (using the notations of section 5). The simulation consists of a finite-difference semi-discretization in space with 100 cells, the resulting set of ODEs being integrated with a stiff implicit solver (Simulink ode15s); as expected $\hat{\theta}$ closely agrees with the truncated series $\bar{\theta}$. 


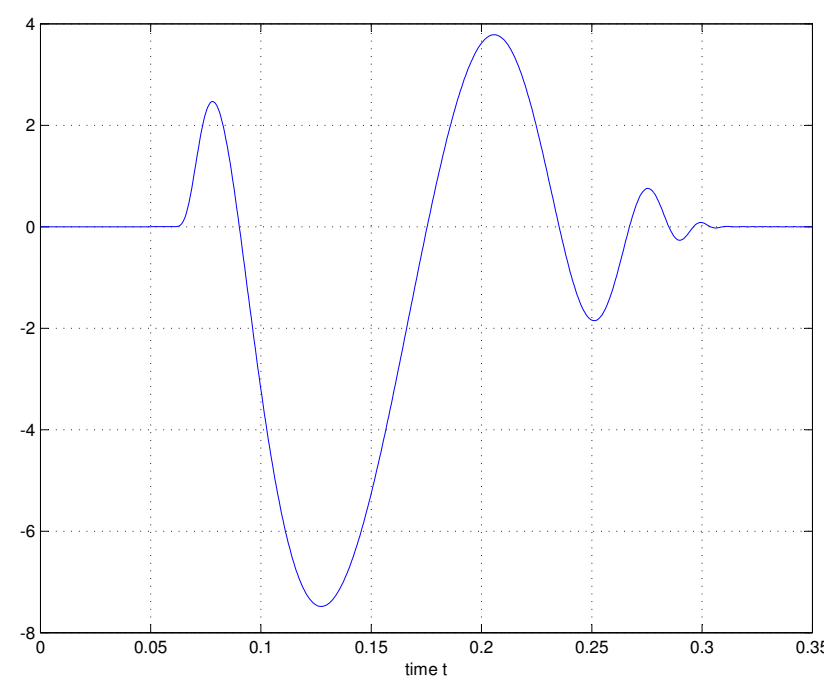

Figure 1. 1D case: time evolution of the control $u(t)$.

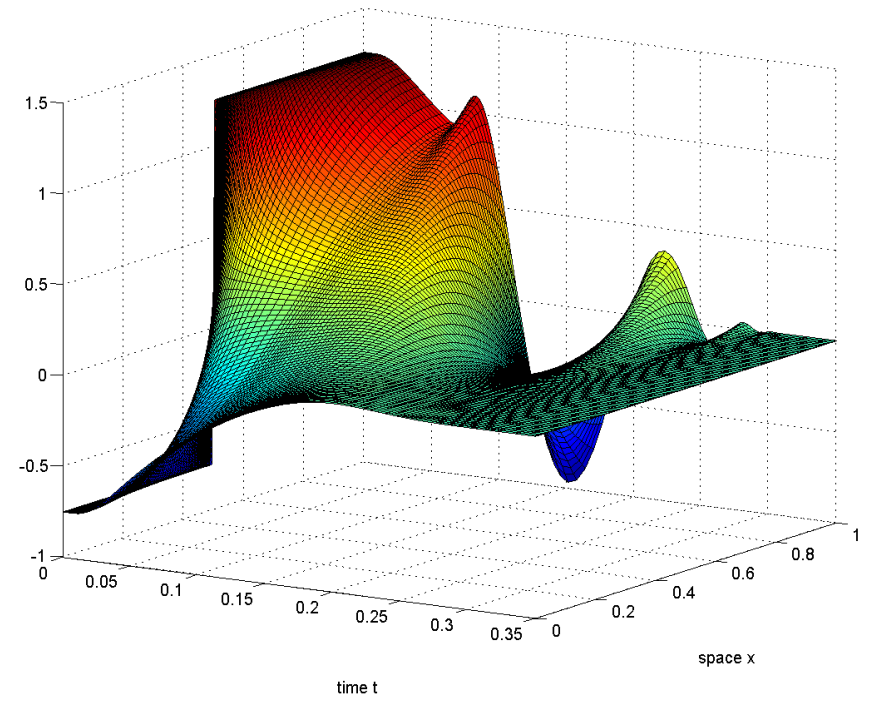

Figure 2. 1D case: time evolution of the temperature $\theta(t, x)$.

\subsection{Example in dimension 2}

$T, \tau, s, \bar{\imath}, \bar{n}$ are the same as previously, with moreover $\bar{\jmath}=$ 25 and $L=1$. The initial condition is the "double step"

$$
\theta_{0}\left(x_{1}, x_{2}\right):= \begin{cases}-1 & \text { if }\left(x_{1}, x_{2}\right) \in\left(0, \frac{1}{2}\right) \times\left(0, \frac{1}{2}\right), \\ 1 & \text { if }\left(x_{1}, x_{2}\right) \in\left(0, \frac{1}{2}\right) \times\left(\frac{1}{2}, 1\right) \\ 1 & \text { if }\left(x_{1}, x_{2}\right) \in\left(\frac{1}{2}, 1\right) \times\left(0, \frac{1}{2}\right), \\ -1 & \text { if }\left(x_{1}, x_{2}\right) \in\left(\frac{1}{2}, 1\right) \times\left(\frac{1}{2}, 1\right) .\end{cases}
$$

Its nonzero Fourier coefficients are

$$
c_{2 l+1,2 p+1}=-2 \frac{(-1)^{l+p}}{\pi^{2}(2 l+1)(2 p+1)}, \quad l, p \geq 0 .
$$

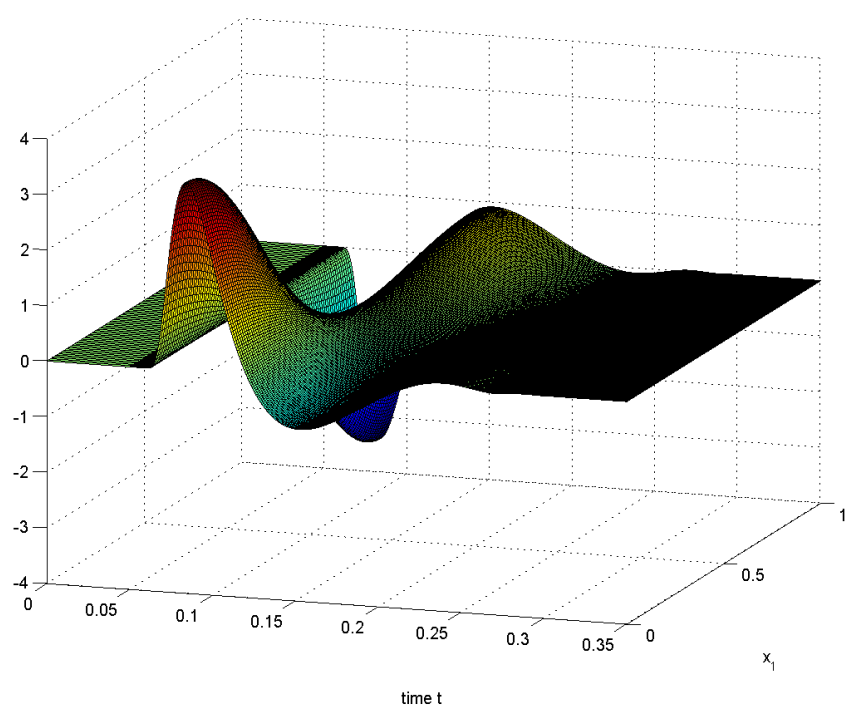

Figure 3. 2D case: time evolution of the control $u\left(t, x_{1}\right)$.

Fig. 3 shows the control $u\left(t, x_{1}\right)$ given by Theorem 8 .

\section{References}

[1] F. Belgacem and S. Kaber. On the dirichlet boundary controllability of the one-dimensional heat equation: Semianalytical calculations and ill-posedness degree. Inverse Problems, 27(5):055012, 2011.

[2] F. Boyer, F. Hubert, and J. Le Rousseau. Uniform controllability properties for space/time-discretized parabolic equations. Numer. Math., 118(4):601-661, 2011.

[3] C. Carthel, R. Glowinski, and J. Lions. On exact and approximate boundary controllabilities for the heat equation: A numerical approach. Journal of Optimization Theory and Applications, 82(3):429-484, 1994.

[4] H. Fattorini and D. Russell. Exact controllability theorems for linear parabolic equations in one space dimension. Arch. Rational Mech. Anal., 43(4):272-292, 1971.

[5] E. Fernández-Cara and A. Münch. Numerical null controllability of the 1D heat equation: primal methods. HAL preprint http://hal.archives-ouvertes.fr/hal-00687884, 2011.

[6] M. Fliess, J. Lévine, P. Martin, and P. Rouchon. Flatness and defect of non-linear systems: Introductory theory and examples. International Journal of Control, 61(6):1327-1361, 1995.

[7] A. V. Fursikov and O. Y. Imanuvilov. Controllability of evolution equations, volume 34 of Lecture Notes Series. Seoul National University Research Institute of Mathematics Global Analysis Research Center, 1996.

[8] G. Garcia, A. Osses, and M. Tapia. A heat source reconstruction formula from single internal measurements using a family of null controls. Journal of Inverse and IIIposed Problems, to appear.

[9] D. Gilbarg and N. Trudinger. Elliptic partial differential equations of second order. Classics in Mathematics. SpringerVerlag, Berlin, 2001. Reprint of the 1998 edition.

[10] L. Hörmander. The analysis of linear partial differential operators. I. Springer-Verlag, 1983. 
[11] B. Jones Jr. A fundamental solution for the heat equation which is supported in a strip. J. Math. Anal. Appl., 60(2):314324, 1977.

[12] B. Laroche, P. Martin, and P. Rouchon. Motion planning for the heat equation. Int J Robust Nonlinear Control, 10(8):629643, 2000.

[13] G. Lebeau and L. Robbiano. Contrôle exact de l'équation de la chaleur. Comm. Partial Differential Equations, 20(12):335-356, 1995.

[14] Y.-J. Lin Guo and W. Littman. Null boundary controllability for semilinear heat equations. Appl Math Optim, 32(3):281316, 1995.

[15] W. Littman. Boundary control theory for hyperbolic and parabolic partial differential equations with constant coefficients. Ann. Scuola Norm. Sup. Pisa Cl. Sci. (4), 5(3):567-580, 1978.

[16] W. Littman and S. Taylor. The heat and Schrödinger equations: boundary control with one shot. In Control methods in PDE-dynamical systems, volume 426 of Contemp. Math., pages 293-305. Amer. Math. Soc., Providence, RI, 2007.

$[17]$ W. A. J. Luxemburg and J. Korevaar. Entire functions and Müntz-Szász type approximation. Trans. Amer. Math. Soc., 157:23-37, 1971.

[18] A. Lynch and J. Rudolph. Flatness-based boundary control of a class of quasilinear parabolic distributed parameter systems. Int J Control, 75(15):1219-1230, 2002.

[19] P. Martin, L. Rosier, and P. Rouchon. Null controllability of the 1D heat equation using flatness. In 1st IFAC workshop on Control of Systems Governed by Partial Differential Equations (CPDE2013), 2013.

[20] P. Martin, L. Rosier, and P. Rouchon. Null controllability of the $2 \mathrm{D}$ heat equation using flatness. In 52nd IEEE Conference on Decision and Control, 2013.

[21] T. Meurer. Flatness-based trajectory planning for diffusionreaction systems in a parallelepipedona spectral approach. Automatica, 47(5):935-949, 2011.

[22] T. Meurer and M. Zeitz. Model inversion of boundary controlled parabolic partial differential equations using summability methods. Math. Comput. Model. Dyn. Syst., 14(3):213-230, 2008.

[23] S. Micu and E. Zuazua. Regularity issues for the nullcontrollability of the linear 1-d heat equation. Syst Control Lett, 60(6):406-413, 2011.

[24] A. Münch and E. Zuazua. Numerical approximation of null controls for the heat equation: ill-posedness and remedies. Inverse Problems, 26(8):085018, 39, 2010.

[25] J.-P. Ramis. Dévissage Gevrey. In Journées Singulières de Dijon (Univ. Dijon, Dijon, 1978), volume 59 of Astérisque, pages 4, 173-204. Soc. Math. France, Paris, 1978.

[26] J. Roe. Elliptic operators, topology and asymptotic methods, volume 395 of Pitman Research Notes in Mathematics Series. Longman, Harlow, second edition, 1998.

[27] L. Rosier. A fundamental solution supported in a strip for a dispersive equation. Comput. Appl. Math., 21(1):355-367, 2002. Special issue in memory of Jacques-Louis Lions.

[28] W. Rudin. Real and complex analysis. McGraw-Hill Book Co., third edition, 1987.

[29] M. A. Shubin. Pseudodifferential operators and spectral theory. Springer-Verlag, Berlin, second edition, 2001.

[30] T. Yamanaka. A new higher order chain rule and gevrey class. Annals of Global Analysis and Geometry, 7(3):179-203, 1989.
[31] C. Zheng. Controllability of the time discrete heat equation. Asymptotic Anal, 59(3-4):139-177, 2008.

[32] E. Zuazua. Control and numerical approximation of the wave and heat equations. In International Congress of Mathematicians. Vol. III, pages 1389-1417. Eur. Math. Soc., Zürich, 2006. 